\title{
A Methodology to Evaluate the Effectiveness of Traffic Balancing Algorithms ${ }^{\star}$
}

\author{
J.E. Villalobos ${ }^{1}$, J.L. Sánchez ${ }^{1}$, J.A. Gámez ${ }^{1}$, J.C. Sancho ${ }^{2}$, and A. Robles ${ }^{2}$ \\ 1 Dept. de Informática \\ Universidad de Castilla-La Mancha \\ 02071- Albacete, Spain \\ \{jvillalobos, jsanchez, jgamez\}@info-ab.uclm.es \\ 2 Dept. de Informática de Sistemas y Computadores \\ Universidad Politécnica de Valencia \\ 46071- Valencia, Spain \\ \{jcsancho, arobles\}@gap.upv.es
}

\begin{abstract}
Traffic balancing algorithms represent a cost-effective alternative to balance traffic in high performance interconnection networks. The importance of these algorithms is increasing since most of the current network technologies for clusters are either based on source routing or use deterministic routing. In source-routed networks, the host is responsible for selecting the suitable path among the set of paths provided by the routing algorithm. The selection of an optimal path that maximizes the channel utilization is not trivial because of the huge amount of combinations. Traffic balancing algorithms are based on heuristics in order to find an optimal solution. In this paper, we propose a new methodology based on the use of metaheuristic algorithms to evaluate the effectiveness of traffic balancing algorithms. Preliminary results show that the set of paths provided by current traffic balancing algorithms are still far from an optimized solution. Thus, it is worth continuing to design more efficient traffic balancing algorithms.
\end{abstract}

\section{Introduction}

Clusters of PCs represent a cost-effective alternative to parallel computers. The use of high performance switch-based interconnects, such as Myrinet [1], Gigabit Ethernet [2], and InfiniBand [3], provides the flexibility, low latency and high bandwidth required in these environments. Often, the interconnection pattern between switches is defined by the user, which may be irregular. To manage such an irregularity, generic routing algorithms can be used, such as $u p^{*} /$ down ${ }^{*}[4]$, smart [5], adaptive-trail [6], and minimal adaptive [7]. $\mathrm{Up}^{*} / \mathrm{down}^{*}$ is the most popular routing algorithm used in clusters. However, this algorithm imposes a large number of routing restrictions in order to remove cyclic channel dependencies in the network graph, which prevent most of the messages from being

* This work was partly supported by the Spanish CICYT under Grant TIC2003-08154C06 and JCC de Castilla La-Mancha under Grants PBC-02-008 and PBC-02-002. 
routed through minimal paths. Unfortunately, this fact has a negative impact on the traffic balance since it tends to concentrate the traffic in a few channels, which dramatically limits its performance.

In order to balance the traffic in the network, dynamic or static strategies can be applied. Dynamic strategies select the routing paths depending on the network status. This status can be known either locally (adaptive routing strategies $[8],[9],[10]$ ) or globally (source-based routing schemes [11]). However, current commercial interconnects do not support adaptive routing mainly due to the possible increase in switch complexity and the difficulties in guaranteeing in order packet delivery. In addition, acquiring global information about the network status can introduce high overhead in the network due to the generated control traffic. Therefore, dynamic strategies to balance traffic are not suitable for application to clusters of PCs.

On the other hand, unlike dynamic strategies, static strategies do not require any hardware support. Thus, they can be applied to any commercial interconnect. These strategies try to achieve an even routing path distribution among the network channels to maximize the network utilization. To this end, traffic balancing algorithms can be used. These algorithms focus on selecting only one path between every source-destination pair, without taking into account the network status. Traffic balancing algorithms select each routing path among the set of paths provided by the applied routing algorithm. Usually, these algorithms assume a uniform packet destination distribution due to the fact that the traffic pattern cannot be known in advance. The use of these algorithms is suitable in networks that either apply source routing [1] or where the routing tables at switches provide deterministic routing [3]. Moreover, unlike adaptive routing strategies, traffic balancing algorithms guarantee the message delivery in order, which is required by many parallel applications.

Traffic balancing algorithms are based on an iterative procedure by which the routing paths provided by the applied routing algorithm are progressively discarded until only one routing path remains between each source-destination pair. Different heuristics can be applied to discard routing paths. The simplest criterion is the random selection. Its main drawback is that it cannot guarantee an even distribution of routing paths over the network channels. More efficient algorithms have been proposed in the literature, such as Summatory of Crossing paths [1], Deviation of Crossing paths [12], and Maximum Crossing path [13].

Given that these algorithms are based on an iterative procedure, it is clear that their effectiveness will strongly depend on the order in which routing paths are discarded. In this sense, we wonder whether these algorithms are able to obtain an optimal solution that achieves the highest performance within the bounds imposed by the applied routing scheme. To answer this question, we should firstly try to obtain the optimal solution. To this end, it would be necessary to evaluate all the possible combinations among the routing paths provided by the applied routing algorithm. However, this approach is non-viable in terms of computational time due to the huge amount of possible combinations. 
An alternative to the enumeration of all the possible combinations, is the use of a heuristic algorithm. In this way, we will be sure to obtain a good solution (possibly a local optima) to the problem in a reasonable time. Concretely, we propose to use metaheuristic algorithms [14] because they can be applied to a wide set of different optimization problems, requiring less effort to adapt them to a specific problem. Nowadays, metaheuristics are widely used to solve important practical combinatorial optimization problems, and so we think that this can be an attractive approach to tackle with the problem posed in this work.

In this paper, we propose a new methodology to evaluate the effectiveness of traffic balancing algorithms, which is based on the use of metaheuristic techniques. This evaluation methodology allows us to know at what extent the performance provided by current traffic balancing algorithms differs from that provided by the optimal solution.

The rest of this paper is organized as follows. In Section 2 we describe the traffic balancing algorithm selected in the evaluation of the new methodology. Section 3 briefly describes the metaheuristics used in this paper. Section 4 describes the methodology followed in the evaluation and Section 5 shows some preliminary results achieved. And finally, Section 6 provides some concluding remarks and indications on future research.

\section{Computing Routing Tables}

We can identify three main stages in the routing table computation process. First, all the shortest paths between every source-destination pair are computed. To this end, the routing algorithm must be able to provide several routing paths between every pair of nodes. Secondly, a traffic balancing algorithm will be applied to select a unique routing path for every source-destination pair. Finally, routing tables must be filled.

To illustrate the application of the proposed evaluation methodology, we have selected the up*/down* routing algorithm, and the Maximum Crossing Path (MaxCp) [13] traffic balancing algorithm. Up*/down* routing is able to provide several paths between every pair of nodes, especially when the DFS methodology [15] is applied to compute its routing paths. Moreover, MaxCp achieves better performance than other traffic balancing algorithms at a lower computation cost when using only a virtual channel.

The MaxCp algorithm is based on minimizing the crossing-path metric, which represents the number of routing paths crossing each channel (i.e., the channel utilization). This algorithm tries to achieve a uniform channel utilization, preventing a few channels from becoming a bottleneck in the network. To this end, the algorithm associates a counter to every channel in the network. Each counter is initialized to the value of the crossing-path metric. Next, a procedure is repetitively applied to the channel with the highest counter value. In each step, a path crossing the channel is selected to be removed if there is more than one path between the source and the destination nodes of that routing path, thus preventing the network from becoming disconnected. When a routing path is removed, the 
counters associated with every channel crossed by the path are updated. Among the routing paths belonging to a channel, the algorithm will firstly choose the path whose source and destination hosts have the highest number of routing paths between them. The algorithm finishes when the number of routing paths between every pair of nodes is reduced down to the unit.

\section{Metaheuristics}

As defined in the Metaheuristics Network ${ }^{1}$ home page, " a metaheuristic is a set of concepts that can be used to define heuristic methods that can be applied to a wide set of different problems". That is, a metaheuristic can be viewed as a general algorithmic framework which allows us to apply a combinatorial optimization technique to a great variety of problems, with relatively few modifications when instancing the method to a particular problem. In this work, we have selected two metaheuristics which belongs to two different approaches: genetic algorithms (from evolutionary computation) and simulated annealing (from the neighborhood search approach).

\subsection{Genetic Algorithms}

Genetic algorithms (GAs) ([16], [14] chapter 3) are evolutionary algorithms based on the principles of natural evolution. GAs try to evolve a population of individuals (potential solutions to the problem) during the search process. Evolution is based on the application of genetic-based operators as selection, crossover and mutation to the individuals contained in the population. A GA works in three main steps:

1. Selection: some individuals are selected from the current population by considering their fitness (the goodness of the solution they codify). Different strategies can be used: proportional to fitness, rank-based, tournament, etc.

2. Reproduction: the selected individuals are grouped (married) in pairs and some offsprings are obtained by crossover, that is, by interchanging genetic material between the parents. For example, from aaaaaa and bbbbbb we obtain aabbbb and bbaaaa when position two is selected as the crossover point. After crossover, some individuals are mutated (slightly changed) in order to introduce some diversity in the search. For example, following our example, aabbab corresponds to mutate position five of the first offspring.

3. Replacement: The new population/generation is obtained by selecting the best individuals from the old and new ones.

This process (steps 1 to 3 ) is repeated until a certain termination condition is achieved. In this work we use Steady State GAs, a model of GA in which only a pair of individuals is selected for reproduction in step 1 . The good behavior of this model has been largely demonstrated in the specific literature.

\footnotetext{
${ }^{1}$ http://www.metaheuristics.org
} 


\subsection{Simulated Annealing}

Simulated Annealing (SA) ([17], [14] chapter 10) is probably the oldest metaheuristic known and has its origin in statistical mechanics. In SA instead of maintaining a population of solutions, only a solution is considered at each state, being its neighborhood explored at each iteration.

SA behaves as a hill climbing algorithm, that is, given the current solution $s$, the algorithm moves to the best solution selected among the neighborhood $(N(s))$ of $s$ (a neighbor $s^{\prime}$ is a solution obtained from $s$ by making a small change or movement). The key idea in SA is to allow moves resulting in worse solutions than the current one in order to avoid being trapped in local optima (as usually happens in hill climbing). To implement this behavior, a parameter $T$ called temperature is used. Concretely, the probability of accepting a neighbor $s^{\prime}$ of $s$ is computed following the Boltzmann distribution: $\exp \left(-\frac{f\left(s^{\prime}\right)-f(s)}{T}\right)$. Therefore, when $T$ is high, the probability of accepting a worse configuration is also high, but when the value of $T$ decreases, the probability of accepting a worse solution also decreases. The value of $T$ is decreased/cooled during the search process by following a cooling schedule.

\section{Methodology of Evaluation}

The methodology proposed to evaluate the effectiveness of the traffic balancing algorithms is based on the two metaheuristics previously described. As has been mentioned, the goal of these algorithms is to provide a high quality (the optimal one ideally) solution from a set of possibles configurations. In the problem of evaluating the effectiveness of traffic balancing algorithms, the optimal solution corresponds to the set of paths which achieve the highest performance in the network when considering only a unique route for every source-destination node pair. We will use the solution found by the metaheuristics to evaluate the effectiveness of the traffic balancing algorithm described in Section 2.

Below we will specify the parameter choices for both metaheuristic algorithms, but first let us to introduce two crucial points which are common to them: individual representation and evaluation.

- Individual representation. An individual will be a vector of integers, where each position corresponds to a pair of (source, destination) nodes, and its value is in the range $2 . . k$, being $k$ the number of different paths from source to destination. Pairs having only a possible route between them are not included in the solution. So, the representation length is actually considerably smaller than the upper bound $n(n-1)$, with $n$ the number of nodes in the network.

- Individual evaluation. Due to the large number of individuals to be evaluated during the search process, the use of a simulator, although very precise, is prohibitive in terms of CPU time. As an alternative we propose to use a performance metric, which can be easily computed from the routing paths codified by the individual. In our initial experiments we have tried with several routing metrics, as minimizing the maximum use of a channel, the 
deviation in channels use, and the sum of channels use. Among them, we have selected the minimization of the deviation in channel use, because it is the one which achieves the results closer to the simulator use.

\subsection{Genetic Algorithm Design}

In order to completely specify the Steady State GA we have to consider the following parameters:

- Initial Population. Individuals in the initial population are generated at random. - Selection. We use rank-based selection. That is, individuals are sorted according to its fitness and each individual receives a probability of selection proportional to its position in the ranking. This mechanism of selection helps to avoid premature convergence.

- Crossover operator. Two offsprings are generated by using the classical onepoint crossover.

- Mutation operator. Each position of each individual in the population is selected for mutation with a small probability (0.01 or 0.02 in our case).

- Stopping Criterion. The algorithm is allowed to complete a fixed number of generations which depends on the network size.

\subsection{Simulated Annealing Design}

The main point here is to define the neighborhood of a given solution $s$. In our case, $s^{\prime}$ it is said a neighbor of $s$ if they differ in exactly one position. That is, given $s=$ aaaaaa, then abaaaa or aaaaba are in $N(s)$, but abaaba is not a neighbor of $s$. On the other hand, as in our GA design, the initial solution is randomly generated and a maximum number of iterations is considered as stopping criterion. The remaining parameters/decisions are:

- Iterations per Temperature. The number of iterations to be carried out before to modify the temperature is the minimum between 1000 and $\prod_{i=1}^{m} k_{i}$, being $m$ the individual length and $k_{i}$ the number of different paths between the pair (sourcedestination) associated with position $i$. In this way we allow the algorithm to carry out a high number (in function of the network complexity) of iterations per temperature, in order to achieve a stable behavior.

- Cooling schedule. The temperature decreases geometrically by using the expression: $T_{i+1}=\alpha T_{i}$, with $\alpha=0.95$. This is without any doubt the most popular cooling scheme in simulated annealing, because it allows a fast decreasing during the exploration phase and a slow cooling during the exploitation phase.

\section{Performance Evaluation}

In this section, we evaluate by simulation the performance of the optimal routing path configuration resulting from applying the metaheuristic algorithm proposed in Section 4 and compare it with that of the MaxCp traffic balancing algorithm. 
We use the up*/down* routing algorithm based on the DFS methodology to compute the routing tables. To obtain realistic simulation results, we have used timing parameters for the switches taken from a commercial network. We have selected Myrinet because it is becoming increasingly popular due to its good performance/cost ratio. Myrinet uses source routing. Packet sizes of 32 and 1024 bytes are used. Also, we assume a uniform distribution of packet destinations. Traffic injection rate is the same for all the hosts. As shown by many works, uniform distribution can be considered the most suitable synthetic traffic pattern for analyzing interconnection networks under general purpose applications exhibiting undetermined and variable traffic patterns with different locality degrees.

\subsection{Network Model}

The network is composed of a set of switches and hosts, all of them interconnected by links. Irregular network topologies are considered in the evaluation. The topologies have been generated randomly. We have generated ten different topologies for each network size analyzed. Results in this paper correspond to the topologies that achieve the average behavior for each network size.

Each switch has 8 ports wherein 4 ports connect to hosts and the remainder connect to other switches. Network sizes of $8,16,32$, and 64 switches have been considered in order to evaluate the influence of the network size on performance. These network sizes can be considered representative of clusters of PCs used nowadays. Note that connecting 4 hosts per switch, a 64-switch network would support up to 256 hosts.

We assume short LAN cables to interconnect switches and hosts. These cables are 10 meters long, offer a bandwidth of $16 \mathrm{MB} / \mathrm{s}$, and have a delay of $4.92 \mathrm{~ns} / \mathrm{m}$. Flits are one byte wide. Physical channel is also one flit wide. Transmission of data across channels is pipelined. Hence, a new flit can be injected into the physical channel every $6.25 \mathrm{~ns}$ and there can be a maximum of 8 flits on the link at a given time. A hardware stop and go flow control protocol [1] is used to prevent packet loss. The first flit latency through the switch is $150 \mathrm{~ns}$.

\subsection{Simulation Results}

For the sake of brevity, the graphs showed in this paper correspond to a 32switch network that exhibits an average behavior. The results for the remainder are qualitatively similar. Figure 1 shows the average latency for the metaheuristic algorithm (MetaHe) and MaxCp traffic balancing algorithm (MaxCp) when short (a) and long (b) packets are used. As can be seen, the routing path configuration obtained by applying the metaheuristic algorithm achieves better performance than the one selected by applying the MaxCp traffic balancing algorithm. The difference in throughput is roughly a 20 percent, regardless of the network size and the packet length used. We have also analyzed bimodal traffic under different rates of short and long messages, obtaining a behavior very similar, in relative terms, to that exhibited with only short or long messages. 
As the performance of the MaxCp algorithm is higher than that of other existing algorithms, the results obtained in this evaluation can be generalized to other low-performance algorithms. These results show that there is still enough margin to improve the performance of traffic balancing algorithms. Notice that the performance improvements resulting from applying traffic balancing algorithms are achieved without requiring any hardware support that would increase the overall system cost. Therefore, these algorithms represent a cost-effective approach to improve the performance of current commercial interconnects.

On the other hand, despite the fact that metaheuristic algorithms often allow us to obtain a set of routing paths able to provide an optimal traffic balance, they would not be advisable to use them in their own right, rather than for benchmarking other algorithms. The reason is that metaheuristic algorithms exhibit a high computation cost which may not be acceptable in most cluster environments. The execution time of the applied metaheuristic algorithm depends on the network size. It ranges between one hour and one hour and a half for 8-switch and 64-switch networks, respectively, when running on a Myrinet cluster of 16 PCs (350 MHz Pentium III with 128 Mbytes RAM).
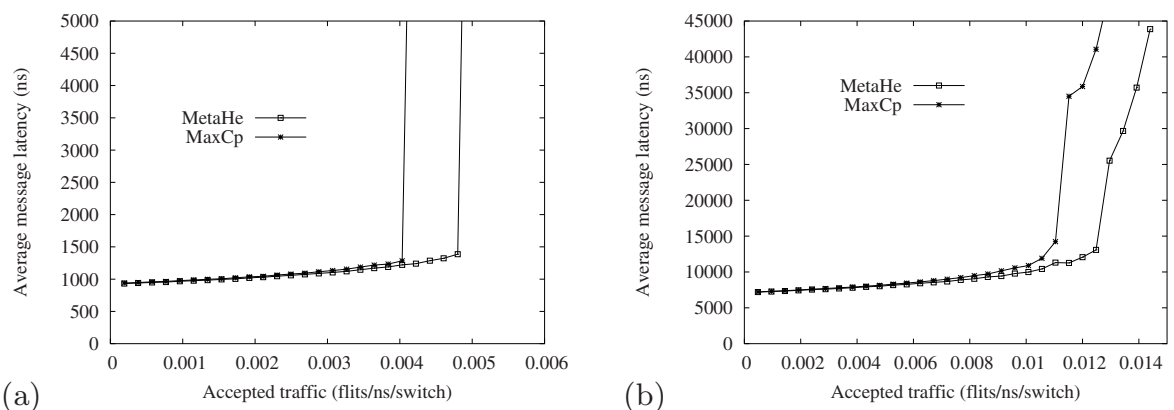

Fig. 1. Average message latency vs accepted traffic for a 32-switch network. Message length is (a) 32 bytes and (b) 1024 bytes.

\section{Conclusions}

In this paper, we proposed a new methodology to evaluate the effectiveness of traffic balancing algorithms. This methodology is based on comparing the performance achieved by the set of paths selected by applying a traffic balancing algorithm against that achieved by the set of routing paths selected by using a metaheuristic algorithm.

Preliminary results obtained for a representative traffic balancing algorithm have shown that its performance is significantly lower than that obtained by using a metaheuristic algorithm. In particular, a difference in throughput as high as 20 percent is obtained. However, the metaheuristic algorithms cannot guarantee that in all the configurations they will always provide an optimized set 
of routing paths that achieve the best performance, and can even be detrimental to performance. These results encourage us to continue to searching for more effective traffic balancing algorithms suitable to improve the performance of current commercial interconnects.

For future work, we plan to analyze the causes that limit the performance of current traffic balancing algorithms in order to find new criterions to select the final set of routing paths. The resulting algorithms must be viable in terms of computational time. Also, we plan to study the effectiveness of traffic balancing algorithms in the context of regular networks, such as fat trees.

\section{References}

1. Boden, N., Cohen, D., Felderman, R., Kulawik, A., Seitz, C., Seizovic, J., Su, W.: Myrinet - a gigabit per second local area network. IEEE Micro (1995) 29-36

2. Sheifert, R.: Gigabit Ethernet. Addison-Wesley (1998)

3. Association, I.T.: InfiniBand Architecture Specification Vol. 1, Release 1.0. (2000)

4. Schroeder, M., Birrell, A., Burrows, M., Murray, H., Needham, R., Rodeheffer, T., Satterthwate, E., Thacker, C.: Autonet: a high-speed, self-configuring local area network using point-to-point links. IEEE Journal on Selected Areas in Communications 9 (1991) 1318-1334

5. Cherkasova, L., Kotov, V., Rockiki, T.: Fibre channel fabrics: Evaluation and design. In: Procs. of the 29th Hawaii Int. Conference on System Science. (1995)

6. Qiao, W., Ni, L.: Adaptive routing in irregular networks using cut-through switches. In: Procs. of the 1996 Int. Conference on Parallel Processing. (1996)

7. Silla, F., Duato, J.: High-performance routing in networks of workstations with irregular topology. IEEE Trans. on Parallel and Distributed Systems 11 (2000)

8. Dally, W., Aoki, H.: Deadlock-free adaptive routing in multicomputer networks using virtual channels. 4 (1993) 466-475

9. Duato, J.: A new theory of deadlock-free adaptive routing in wormhole networks. 4 (1993) 1320-1331

10. Konstantinidou, S., Snyder, L.: The Chaos router. IEEE Transactions on Computers 43 (1994) 1386-1397

11. Franco, D., Garcés, I., Luque, E.: A new method to make communication latency uniform. In: Procs. of ACM Int. Conference on Supercomputing. (1999) 210-219

12. Flich, J., Malumbres, M., López, P., Duato, J.: Improving routing performance in myrinet networks. In: Procs. of the Int. Parallel and Distributed Processing Symposium. (2000)

13. Sancho, J., Robles, A.: Improving the up*/down* routing scheme for network of workstation. In: Procs. of the European Conference on Parallel Computing. (2000)

14. Glover, F., Kochenberger, G.: Handbook of Metaheuristics. Kluwer Academic Publishers (2003)

15. Sancho, J., Robles, A., Duato, J.: A new methodology to compute deadlock-free routing tables for irregular topologies. In: Procs. of the 4th Workshop on Communication, Architecture and Applications Network-based Parallel Computing. (2000)

16. Michalewicz, Z.: Genetic Algorithms + Data Structures = Evolution Programs. Springer-Verlag (1996)

17. Laarhoven, P.V., Aarts, E.: Simulated annealing. Reidel Publishing Company (1988) 